\title{
Modeling of Reactor Kinetics and Dynamics
}

Matthew Johnson Scott Lucas Pavel Tsvetkov

September 2010

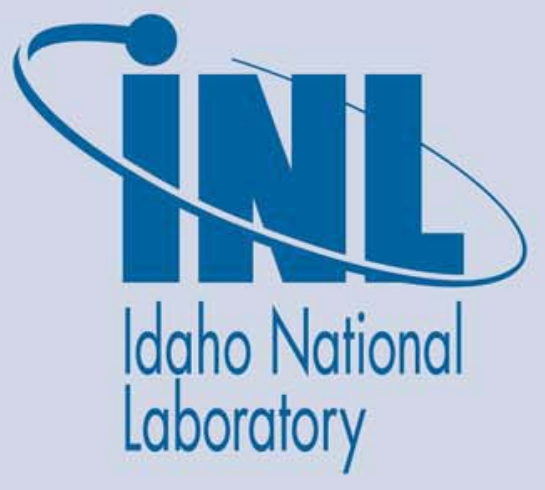

The INL is a U.S. Department of Energy National Laboratory operated by Battelle Energy Alliance 
INL/EXT-10-19953

\title{
Modeling of Reactor Kinetics and Dynamics
}

\author{
Matthew Johnson \\ Scott Lucas \\ Pavel Tsvetkov
}

September 2010

\section{Idaho National Laboratory \\ Idaho Falls, Idaho 83415}

http://www.inl.gov

\author{
Prepared for the \\ U.S. Department of Energy \\ Office of Nuclear Energy \\ Under DOE Idaho Operations Office \\ Contract DE-AC07-05ID14517
}




\title{
Modeling of Reactor Kinetics and Dynamics
}

\author{
Matthew Johnson \\ Scott Lucas \\ Pavel Tsvetkov
}




\section{ABSTRACT}

In order to model a full fuel cycle in a nuclear reactor, it is necessary to simulate the short timescale kinetic behavior of the reactor as well as the long time-scale dynamics that occur with fuel burnup. The former is modeled using the point kinetics equations, while the latter is modeled by coupling fuel burnup equations with the kinetics equations. When the equations are solved simultaneously with a nonlinear equation solver, the end result is a code with the unique capability of modeling transients at any time during a fuel cycle.

\section{Reactor Kinetics}

The traditional use of the point reactor kinetics equations is to model transients over short time periods (seconds to minutes). When the equations are used for this purpose, time dependence in all kinetics parameters other than the reactivity can be safely neglected without introducing significant error into the model [1]. For short time scales where fuel composition changes are negligible, large changes in the delayed neutron fraction, $\beta$, or the mean neutron generation time, $\Lambda$, could not occur without a significant departure from criticality that would invalidate the use of the point kinetics model. For longer time scales, the composition changes that affect the point kinetics equations of the fuel cycle for burnup and transmutation are the subject of this report. In order to examine this behavior, a computer code incorporating reactor kinetics with delayed neutrons and fuel composition changes has been written.

The neutron density and delayed precursor concentrations are obtained from the following equations,

$$
\begin{gathered}
\frac{d n(t)}{d t}=\frac{\rho(t)+\alpha_{F}\left(T_{F}(t)-T_{F, 0}\right)+\alpha_{M}\left(T_{M}(t)-T_{M, 0}\right)-\beta_{m i x}(t)}{\Lambda} n(t)+\sum_{i=1}^{6} \lambda_{i} C_{i}(t)+Q(t) \\
\frac{d C_{i}(t)}{d t}=\frac{\beta_{i}^{\text {mix }}(t)}{\Lambda} n(t)-\lambda_{i} C_{i}(t) \quad, \quad i=1 \ldots 6
\end{gathered}
$$

where $n$ is the neutron density, $\rho$ is reactivity, $T_{F}$ is fuel temperature, $T_{M}$ is moderator temperature, $\beta_{m i x}$ is the effective delayed neutron fraction, $\alpha_{F}$ is the fuel temperature feedback coefficient, $\alpha_{M}$ is the moderator temperature feedback coefficient, $\Lambda$ is the neutron generation time, $C_{i}$ is the group $i$ delayed neutron precursor concentration, $\lambda_{i}$ is the decay constant for the $i$ th delayed neutron precursor group, and $Q$ is an external source of neutrons. 
The point kinetics equations are curious with respect to units. Both reactivity and the delayed neutron fraction are unitless. That is not to say they are arbitrary quantities. Reactivity is a fractional change in the multiplication factor of the system, $\frac{\Delta k}{k}$. The delayed neutron fraction is the fraction of the total neutrons resulting from a fission that are not emitted instantaneously, $\frac{\text { delayed neutrons }}{\text { total neutrons }}$. Furthermore, the temperature feedback coefficients are defined by the following relation

$$
\alpha_{X}=\frac{d \rho}{d T_{X}}
$$

where the $X$ subscript is used to signify either fuel temperature or moderator temperature. When a feedback coefficient is multiplied by a change in temperature the result is a change in reactivity. The only terms in the point kinetics equations with absolute physical units are $\Lambda$ and $\lambda$, which have units of time and inverse time, respectively. In order for the equations to be dimensionally consistent $n$ and $C$ must have the same units, while $Q$ must have units of $\frac{d n}{d t}$. To determine these units, one must look back at the original, more fundamental equations from which point kinetics are derived [2]. The point kinetics equations are derived from diffusion or transport theory- equations that describe the neutron population. The units of $n$ and $C$ must then be $\frac{n}{\mathrm{~cm}^{3}}$ (a density since the point kinetics equations are $0-\mathrm{D}$ ) or any quantity directly proportional to this. In a one-neutron energy group model, the neutron speed $v$ is a constant and therefore the neutron flux, given below in Equation (4), is always directly proportional to the neutron population $n$. This allows the point kinetics equations to be cast in terms of the neutron flux. This is a convenient form of the point kinetics equations as flux is also used calculate reaction rates. For this reason the point kinetics equations are coded in the form given by Equations (5) and (6). The point kinetics equations can also be cast in terms of reactor power.

$$
\begin{gathered}
\phi(t)=v n(t) \\
\frac{d \phi(t)}{d t}=\frac{\rho(t)+\alpha_{F}\left(T_{F}(t)-T_{F, 0}\right)+\alpha_{M}\left(T_{M}(t)-T_{M, 0}\right)-\beta_{\text {mix }}(t)}{\Lambda} \phi(t)+\sum_{i=1}^{6} \lambda_{i} C_{i}(t)+Q(t) \\
\frac{d C_{i}(t)}{d t}=\frac{\beta_{i}^{\text {mix }}(t)}{\Lambda} \phi(t)-\lambda_{i} C_{i}(t) \quad, \quad i=1 \ldots 6
\end{gathered}
$$

For short time scales the time dependence of the fuel and the moderator temperatures are very importance since these determine the feedback for the flux. Additionally, reactivity changes due to control rod motion and boron concentration changes are also relevant. Any additional time 
dependence in any of the parameters in Equations (5) and (6) due to longer-term changes in fuel composition and will be discussed in the section on reactor dynamics.

The fuel temperature is obtained by doing an energy balance on a lumped model for the fuel and clad using Newton's law of cooling. Performing a time-dependent energy balance on the reactor core yields the following relation for the conservation of energy

$$
\frac{d E_{s t}}{d t}=\frac{d E_{g}}{d t}-\frac{d E_{o u t}}{d t}
$$

where $E_{s t}$ is the amount of stored energy in the core, $E_{g}$ is the amount of energy generated in the core, and $E_{\text {out }}$ is the amount of energy removed by the coolant. Equation (7) can now be written as

$$
\rho V c_{p} \frac{d T_{F}}{d t}=P_{e f f}-h A_{s}\left(T_{F}-T_{M}\right)
$$

where $\rho$ is the fuel density, $V$ is the volume of the fuel, $c_{p}$ is the specific heat of the fuel, $P_{\text {eff }}$ is the thermal power of the reactor with corrections for decay heat, $h$ is a convection heat transfer coefficient, $A_{s}$ is the contact area between the fuel and coolant, $T_{F}$ is the fuel temperature, and $T_{M}$ is the moderator temperature. Moving the term $\rho V c_{p}$ over to the right hand side of the equation yields the ordinary differential equation

$$
\frac{d T_{F}}{d t}=\left(\frac{1}{m c_{p}}\right) P_{e f f}-\gamma\left(T_{F}-T_{M}\right)
$$

where $\rho V$ has been combined into $m$, the mass of the fuel, and $\gamma$, which has units of inverse time, is interpreted as time constant characterizing the transfer of heat from the fuel to the moderator [1].

$$
\frac{d T_{M}}{d t}=\text { user defined } f(t)
$$

As shown in Equation (5), the current model does not incorporate time, temperature, or any other dependence into the fuel and moderator temperature feedback coefficients, $\alpha_{F}$ and $\alpha_{M}$. 
Table I

Typical reactivity requirements in a PWR [3]

\begin{tabular}{|l|l|}
\hline Total worth of control, $\Delta \rho$ & 0.32 \\
\hline Control-rod worth & 0.07 \\
\hline Burnable poison worth & 0.08 \\
\hline Chemical shim worth & 0.17 \\
\hline
\end{tabular}

Currently, absorber rods and boron concentration are implemented as the only reactor control mechanisms. Typical worths of the control mechanisms of a PWR of are given in 
Table I. Since burnable poisons have not been implemented in the model yet, their worth was included in the control-rod worth, making the total change in reactivity $(\Delta \rho)$ of the rods 0.15 . A change in reactivity is given by the difference in reactivities over a time step or, for the latest reactivity state defined by the following equation.

$$
\rho^{\prime}=\frac{k^{\prime}-1}{k^{\prime}}
$$

The previous reactivity state is given as:

$$
\rho=\frac{k-1}{k}
$$

The difference in reactivities is given as:

$$
\begin{gathered}
\Delta \rho=\rho^{\prime}-\rho=\frac{k^{\prime}-1}{k^{\prime}}-\frac{k-1}{k} \\
\Delta \rho=\frac{k\left(k^{\prime}-1\right)-k^{\prime}(k-1)}{k k^{\prime}}=\frac{k^{\prime}-k}{k k^{\prime}}
\end{gathered}
$$

If $k$ and $k^{\prime}$ are close to 1.0 , we can use $\Delta \rho=k^{\prime}-k$ for the reactivity change in $\Delta k$. Reactivity insertions are often measured in dollars. The reactivity insertion in dollars is

$$
\Delta \rho(\$)=\left(k^{\prime}-k\right) / \beta
$$

In the interest of simplicity, the integral worth of the control rods was taken to be linear. The exact calculation of the boron worth will be discussed in a later section, but the net result is that boron reactivity worth is also linear.

Control rod position and boron concentration are calculated in the same manner as moderator temperature. The control rod insertion rates and the time rate of change of the boron concentration are given by the user. Alternatively, this formulation could be removed and control rod position and boron concentration could be explicitly input.

$$
\begin{gathered}
\frac{d(\text { control rod })}{d t}=\text { user defined } f(t) \\
\frac{d(\text { boron concentration })}{d t}=\text { user defined } f(t)
\end{gathered}
$$

\section{Reactor Dynamics}


In this paper, the term reactor dynamics refers to changes in reactivity or any of the kinetics parameters that occur on timescales on the order of hours to years. The primary source of these sorts of changes is fuel burnup. Changes in fuel composition over the course of a fuel cycle are taken into account by solving the Bateman relations for 24 different actinides and 4 fission products associated with a commercial PWR or BWR. The Bateman relations are differential equations which track changes in isotopic densities due to nuclear decay and neutron interactions. The general from of these equations is given in Equation (18),

$$
\frac{d{ }_{y}^{x} N}{d t}=\phi(t) *\left({ }_{y}^{x-1} N \sigma_{c}-{ }_{y}^{x} N \sigma_{a}\right)-{ }_{y}^{x} N \lambda+{ }_{*}^{*} N \lambda
$$

where $\phi$ is the scalar flux, $\sigma_{c}$ is the microscopic capture cross section, $\sigma_{a}$ is the microscopic absorption cross section, $\lambda$ is a decay constant, and ${ }_{y}^{x} N$ is the atomic density of isotope $N$ with atomic number $x$ and mass number $y$. The final term of Equation (18), ${ }_{*}^{*} N \lambda$, accounts for gain in $N$ due to the decay of arbitrary isotope ${ }_{*}^{*} N$. For a beta decay ${ }_{*}^{*} N$ would be ${ }_{y-1}^{x} N$, while the format for an alpha decay would be ${ }_{x+2}^{y+4} N$.

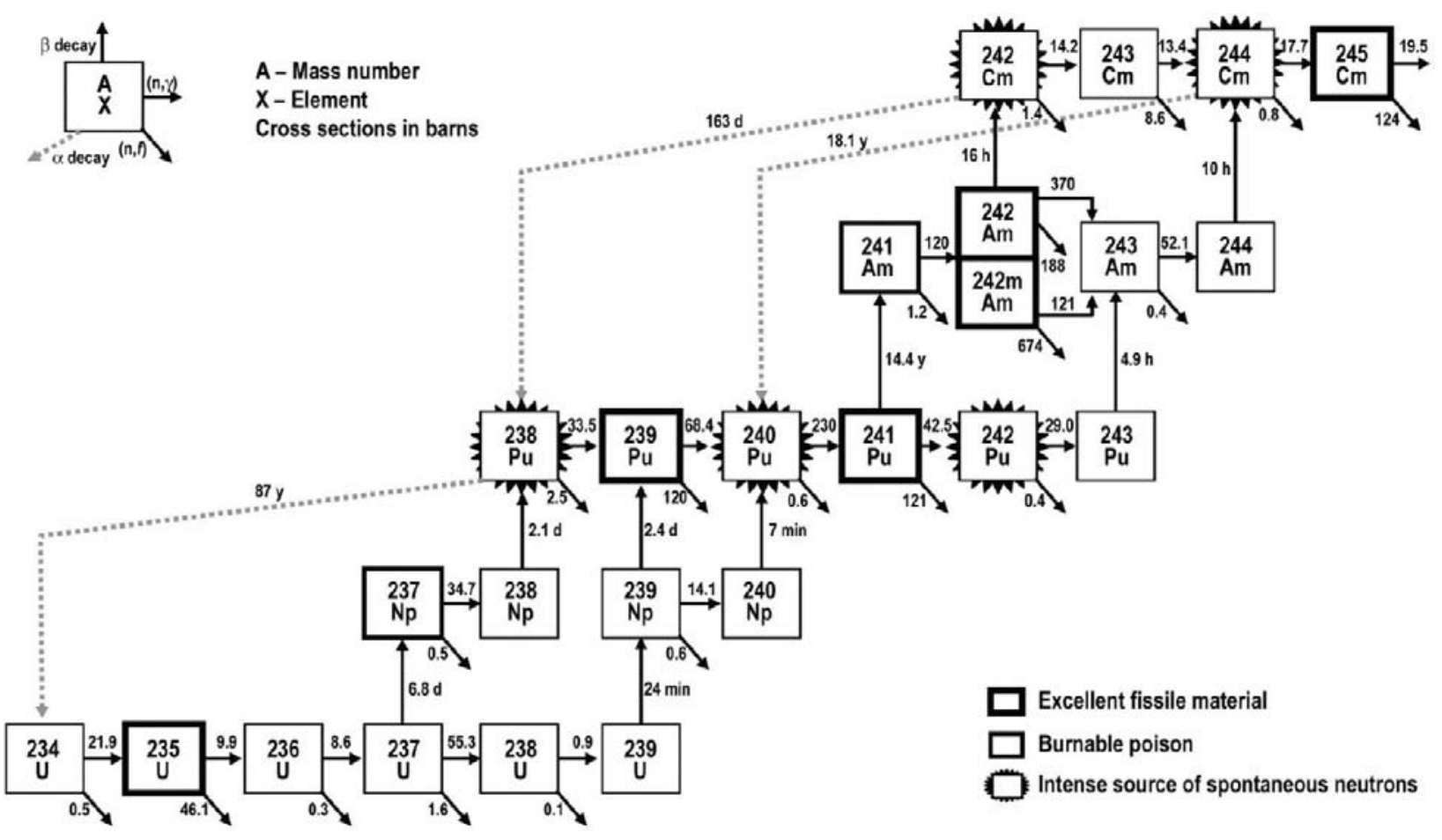

Figure 1. The actinide decay chain currently implemented in the code. Taken from Technology and Applied R\&D Needs for Advanced Nuclear Energy Systems [4]

For example, consider the ${ }^{240} \mathrm{Pu}$ isotope as shown in Figure 1. As source terms, ${ }^{240} \mathrm{Pu}$ arises from neutron capture by ${ }^{239} \mathrm{Pu}$, the beta decay of ${ }^{240} \mathrm{~Np}$, and the alpha decay of ${ }^{244} \mathrm{Cm}$. The only loss 
term considered is neutron absorption. ${ }^{240} \mathrm{Pu}$ has a half-life is 6561 years, so modeling loss from decay is not necessary.

$$
\frac{d_{94}^{240} N}{d t}=\phi(t) *\left({ }_{94}^{239} N \sigma_{c}-{ }_{94}^{240} N \sigma_{a}\right)+{ }_{93}^{240} N \lambda+{ }_{96}^{244} N \lambda
$$

In addition to the actinides, the decay chains of ${ }^{135} \mathrm{Xe}$ and ${ }^{149} \mathrm{Sm}$ are important. ${ }^{135} \mathrm{Xe}$ and ${ }^{149} \mathrm{Sm}$ have very large thermal absorption cross sections making them significant neutron poisons. The equations modeling these fission products take the same form as Eq. (18), only they can include an additional term for production from fission. For example, consider the fission product ${ }^{135} \mathrm{Xe}$ which requires such a term.

$$
\frac{d_{54}^{135} N}{d t}=\phi(t) *\left(0.003 *{ }_{92}^{235} N \sigma_{f}-{ }_{54}^{135} N \sigma_{a}\right)-{ }_{54}^{135} N \lambda+{ }_{53}^{135} N \lambda
$$

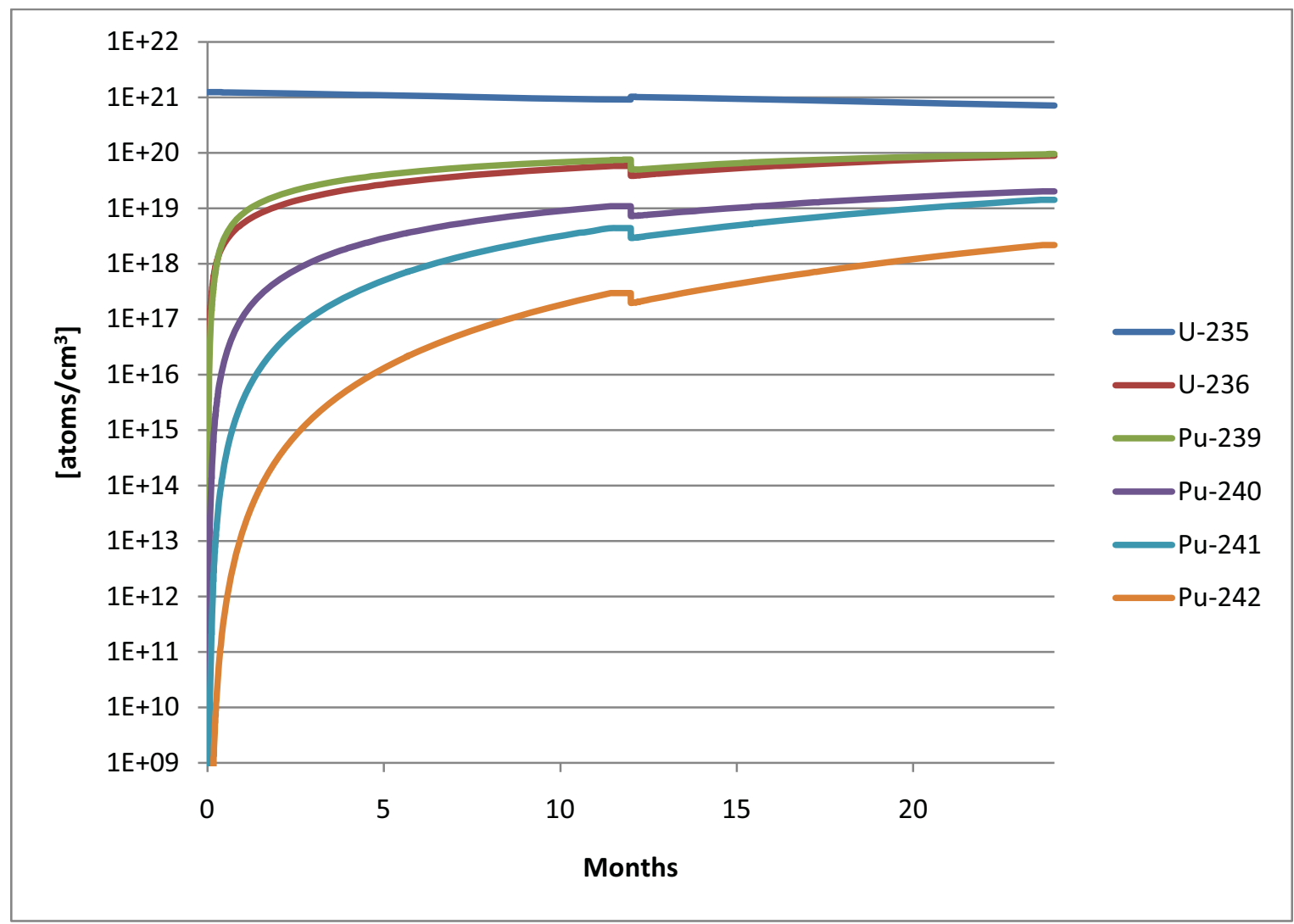

Figure 2. This graph shows example output generated by the depletion equation. The initial fuel at $\mathrm{t}=0$ is completely fresh, so there is no Plutonium content. The reactor is refueled at 12 months, leading to the discontinuities as $\mathrm{Pu}$ concentrations decrease and the ${ }^{235} \mathrm{U}$ concentration increases. 
With the isotopic composition of the fuel vector known, it becomes possible to track the results of fuel burnup on the kinetics parameters, particularly the reactivity $\rho$ and the total effective delayed neutron fraction $\beta$. In order to determine the changes in the reactivity, $\mathrm{k}_{\mathrm{eff}}$ is approximated by Equation (21).

$$
k_{e f f}(t)=\frac{v \Sigma_{f}(t)}{\sum_{a}(t)\left(1+L^{2} B^{2}\right)}
$$

The numerator is calculated summing over the actinide vector, as shown by Equation (22)

$$
v \Sigma_{f}(t)=\sum_{i=1}^{24} v_{i} \sigma_{f, i} N_{i}(t)
$$

The macroscopic absorption cross section must take into account the actinides, the fission products, and any other materials in the reactor which may absorb neutrons, shown in (23).

$$
\Sigma_{a}(t)=\left[\sum_{i=1}^{24} N_{i} \sigma_{a, i}\right]+N_{X e} \sigma_{a}^{X e}+N_{S m} \sigma_{a}^{S m}+F * \sigma_{a}^{\text {fission }}+N_{\text {water }} \sigma_{a}^{\text {water }}+N_{\text {boron }} \sigma_{a}^{\text {boron }}
$$

In the above equation, the first term is a sum over the absorption cross sections due to the actinides. The next two terms calculate the absorption cross sections of ${ }^{135} \mathrm{Xe}$ and ${ }^{149} \mathrm{Sm}$, two fission products of particular interest. The fourth term approximates parasitic absorption for all other fission products. The total number of fissions, $F$, that have occurred in the fuel since the beginning of irradiation, is multiplied by $\sigma_{a}^{\text {fission }}$. Having units of barns per fission, $\sigma_{a}^{\text {fission }}$ is not a true cross-section, but rather a constant of proportionality to convert from total fissions to an absorption macroscopic cross section. The total number of fissions that have taken place is tracked by a simple differential equation that sets $\frac{d F}{d t}$ equal to the fission rate.

$$
\frac{d F}{d t}=\phi(t) \sum_{i=1}^{24} N_{i} \sigma_{f}^{i}
$$

The final terms, $N_{\text {water }} \sigma_{a}^{\text {water }}$ and $N_{\text {boron }} \sigma_{a}^{\text {boron }}$, give the contribution to absorption from the moderator and from boron, respectively. Additional terms could be added to model absorption in structural material.

Once $k_{\text {eff }}$ has been calculated, it is converted to reactivity with the following equation:

$$
\rho(t)=\frac{k_{e f f}-1}{k_{e f f}}
$$


Information on the fuel vector is also used to model the time dependence of the delayed neutron fraction for the fuel mixture, $\beta_{\text {mix }}$. The delayed neutron fraction for ${ }^{239} \mathrm{Pu}$ is about half that of ${ }^{235} \mathrm{U}$. This causes $\beta_{\text {mix }}$ to decrease as ${ }^{235} \mathrm{U}$ burns out and ${ }^{239} \mathrm{Pu}$ builds up. The standard firstestimate method is to use a weighted average set of delayed neutron yields [1] [5] [6]. However, in order to be able to use a weighted average set of $\beta_{\text {mix }}^{i}$ without further adjustment, the delayed neutron data for all three isotopes must be cast to a single set of decay constants. When this is done, the net result is an adjustment in each of the nuclides' $\beta^{i}$. This code uses delayed neutron data that has been fit to the single set of decay constants as given by Ott [7]. Because this data was originally intended for use in fast reactors, the values of $\lambda_{k}$ for all nuclides have been fit to those belonging to ${ }^{239} \mathrm{Pu}$. Ott makes the case that it can also be used in thermal reactors as the differences between $\lambda_{\mathrm{k}}$ for the various nuclides are small.

Since this code models a standard LWR, the isotopes that are considered are ${ }^{235} \mathrm{U},{ }^{239} \mathrm{Pu}$, and ${ }^{238} \mathrm{U}$. The delayed neutron fraction for each delayed neutron group is calculated with the mixture relation:

$$
\beta_{\text {mix }}^{i}=\frac{\sum_{q=1}^{3} v_{q} \sigma_{f}^{q} N_{q}(t) \beta_{q}^{i}}{\sum_{q=1}^{3} v_{q} \sigma_{f}^{q} N_{q}(t)}
$$

Equation (26) weights the group delayed neutron fractions with the rate of neutron production in each fissioning nuclide. The $q$ subscript runs from one to three for the three fissionable nuclides. Other researchers have taken similar approaches [8]. As a final note, a true calculation of the delayed neutron fraction includes taking into account the difference between the energy spectra of prompt and delayed neutrons. Because delayed neutrons are born with less energy, they are more likely to reach thermal energies before leaking or being absorbed. The standard way to account for this is to increase the delayed neutron fraction by an appropriate amount. When this is done, the end result is an effective delayed neutron fraction which, for a large LWR, is couple percent larger than $\beta$ calculated without accounting for the spectral differences [3]. 


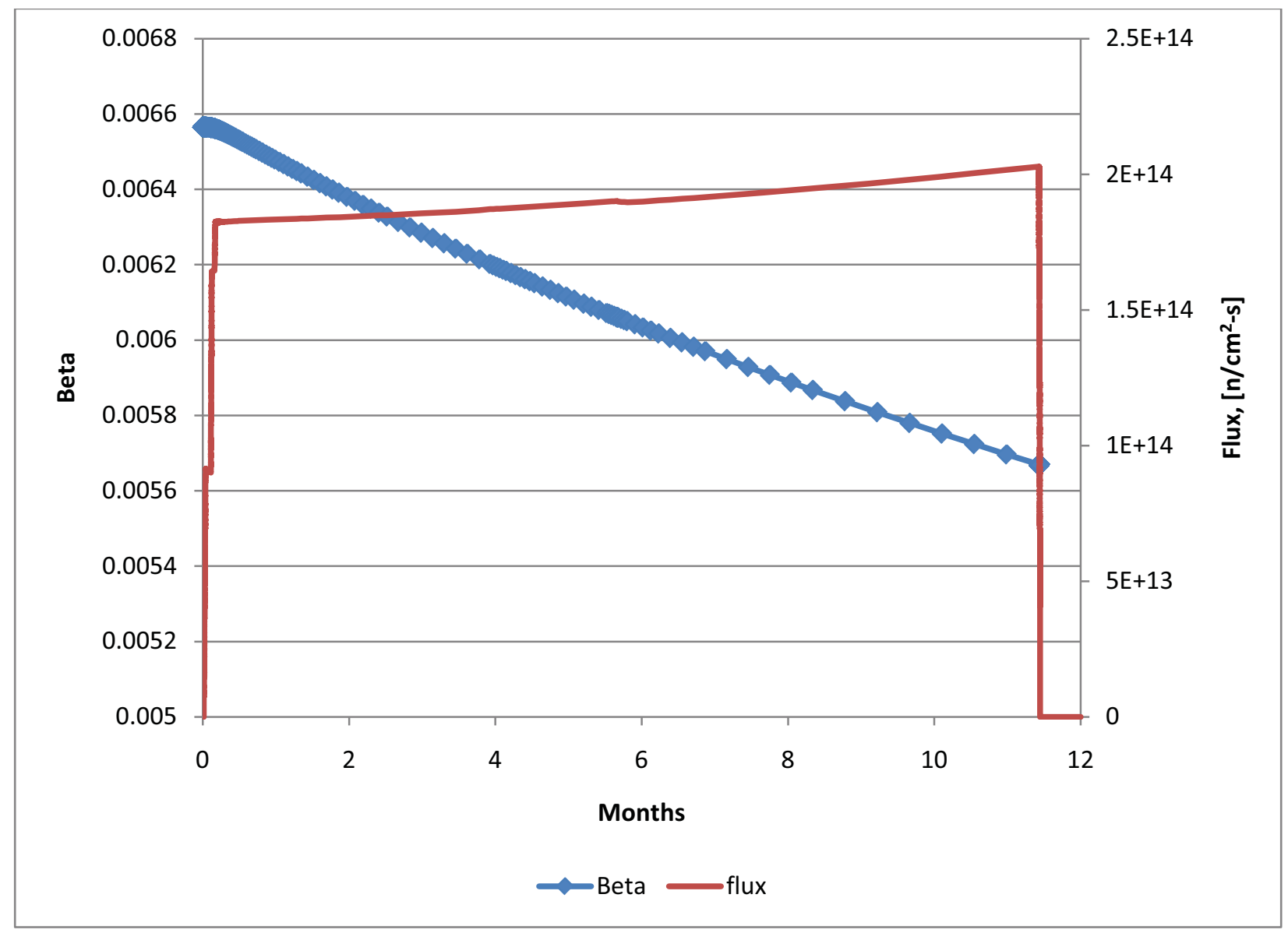

Figure 3. This graph shows the time dependence of the delayed neutron fraction throughout a fuel cycle. The scalar flux in the reactor is also graphed simply to show that the reactor is at power. 


\section{Decay Heat}

Decay heat is the result of the radioactive decay of unstable nuclides. Delayed neutron induced fission is also a source of heat after a reactor has been shut down, but it is not typically classified as "decay heat." The majority of decay heat comes from fission products; however, certain actinides are important for longer decay times. The papers by Tobias and Schrock provide an excellent overview of decay heat in nuclear reactors [10][11]. There are two general ways in which codes calculate decay heat. The first way is to use functions fit to experimental data. The most commonly used functions are the ANS decay heat standards. The second way is to track the isotopic makeup of the fission products present in the fuel and the decay chains of these fission products. This method is computationally intensive, as many different fission products must be tracked. Additionally, such codes must rely on ENDF or similar data for information on fission yields and nuclear decay. ORIGEN [9], a code commonly used in fuel depletion and decay heat calculations, tracks 1119 fission products and the explicit fission product yields for 30 actinides. The paper by Tasaka [12] includes a detailed discussion of the considerations that need to be taken into account when performing decay heat calculations based on tracking fission product generation and decay.

The decay heat model implemented in this code is a partial implementation of the 1979 ANS decay heat standard for LWRs. The optional adjustments that account for decay heat due to actinides and neutron capture in fission products were not implemented. The ANS standard contains information on the decay heat fractions for the three isotopes: ${ }^{235} \mathrm{U},{ }^{238} \mathrm{U}$, and ${ }^{239} \mathrm{Pu}$. The standard consists of exponential decay curves based on experimental and theoretical data. In the interest of conservatism they are biased toward the experiments which yielded the largest values for decay heat, rather than using, for example, a least-squares fit of all experimental data [13]. The ANS-5.1-1979 standard implemented in this code has been superseded by the 2005 revision, ANS-5.1-2005. Code in both TRAC and RETRAN was used as a guide in writing the equations describing decay heat [14][15].

The decay heat equations in their basic form are analogous to the equations for the delayed neutron precursors. They can be thought of as delayed power "concentrations."

$$
\frac{d H_{i j}}{d t}=\frac{E_{i j} P_{i}}{Q}-\lambda_{i j} H_{i j} \quad, \quad i=1 \ldots 3 \quad j=1 \ldots 23
$$

$H_{i j}$ is the delayed power, with units of watts, for group $j$ of isotope $i$. There are 23 delayed power groups for each nuclide. $E_{i j}$ is the delayed power fraction for group $j$ of isotope $i$. These are given by the ANS standard [16]. They have units of $\mathrm{MeV}$ per fission per second. $P_{i}$ is the total power in watts produced by isotope $i$. $Q$ is energy per fission; however, it must have units 
of $\mathrm{MeV}$ per fission in order to be consistent with $E_{i j}$. $\lambda_{i j}$ is the decay constant for group $j$ of isotope $i$, and it too is given by the ANS standard.

The reactor power must be modified to account for decay heat. This is done by introducing $P_{\text {eff }}$, the effective power.

$$
P_{e f f}=P_{i n s t}-\sum_{i=1}^{3} P_{d}^{i}+\sum_{i=1}^{3} \sum_{j=1}^{23} H_{i j}
$$

$P_{i n s t}$ is the instantaneous power [17]:

$$
P_{i n s t}=\sum_{i=1}^{3} \phi E_{f} N_{i} \sigma_{f}^{i} V
$$

This is a traditional power calculation. $E_{f}$ is a measure of the total recoverable energy released by a fission. The majority of this energy is in the kinetic energy of the fission products. The addition factor $V$, the volume of the reactor, must be added because $N_{i}$ has used of [atoms $/ \mathrm{cm}^{3}$ ]. The delayed portion of the power for each isotope, , must also be calculated. This is done by setting $\frac{d H_{i j}}{d t}$ to zero in Eq. (27). Doing this and performing a summation over the $j$ groups of delayed power yields the delayed portion of the power produced by isotope $i$.

$$
P_{d}^{i}=\frac{P_{i n s t}^{i}}{Q} * \sum_{j=1}^{23} \frac{E_{i j}}{\lambda_{i j}}
$$

Note that the delayed fraction is also equal to the long irradiation time equilibrium decay heat for each isotope. For sufficiently long irradiation times at constant power, $P_{\text {eff }}$ will be equal to $P_{\text {inst }}$. Furthermore, the term $\frac{1}{Q} \sum_{j=1}^{23} \frac{E_{i j}}{\lambda_{i j}}$ is the percent of power produced by isotope $i$ that is delayed. These values have been tabulated below. As fuel composition changes with burnup, so does the equilibrium amount of decay heat.

Table II

The table below gives the delayed power percent for each isotope as calculated with data from the ANS decay heat standard.

\begin{tabular}{|l|l|}
\hline & Delayed \% \\
\hline${ }^{235} \mathrm{U}$ & $7.0 \%$ \\
\hline${ }^{239} \mathrm{Pu}$ & $9.7 \%$ \\
\hline${ }^{238} \mathrm{U}$ & $8.2 \%$ \\
\hline
\end{tabular}


Since the isotopic content of the fuel vector is being tracked, there is no need to use the optional adjustment to the ANS decay heat curves that accounts for actinide decay. Instead, the decay power from the actinides can be tracked with the following equation:

$$
P_{N}=Q \lambda N V
$$

where $P_{N}$ is the power in watts produced by the decay of actinide $N, Q$ is the Q-value for the decay with units of $[\mathrm{J} /$ decay $], \lambda$ is the actinide's decay constant, $N$ is the atomic density of the actinide and has units of $\left[\right.$ atoms $\left./ \mathrm{cm}^{3}\right]$, and $V$ is the volume of the reactor.

In an effort to quantify the effects of decay heat of a power excursion, a rapid transient was induced by quick ramp reactivity insertion. The same transient was run twice, once with the decay heat model turned on and once with the decay heat model turned off. With the decay heat model turned off, $P_{\text {eff }}$ is not calculated and $P_{\text {inst }}$ is used instead in Equation (9). Decay heat introduces a time delay in the power output and the scalar flux. Since it is the power output, not the scalar flux, that determines the fuel temperature, decay heat introduces a small lag in the fuel temperature and therefore the Doppler effect. This slight delay in the onset of the Doppler effect gives the prompt neutron population a little more time to grow, causing the peak power to be larger when decay heat is modeled.

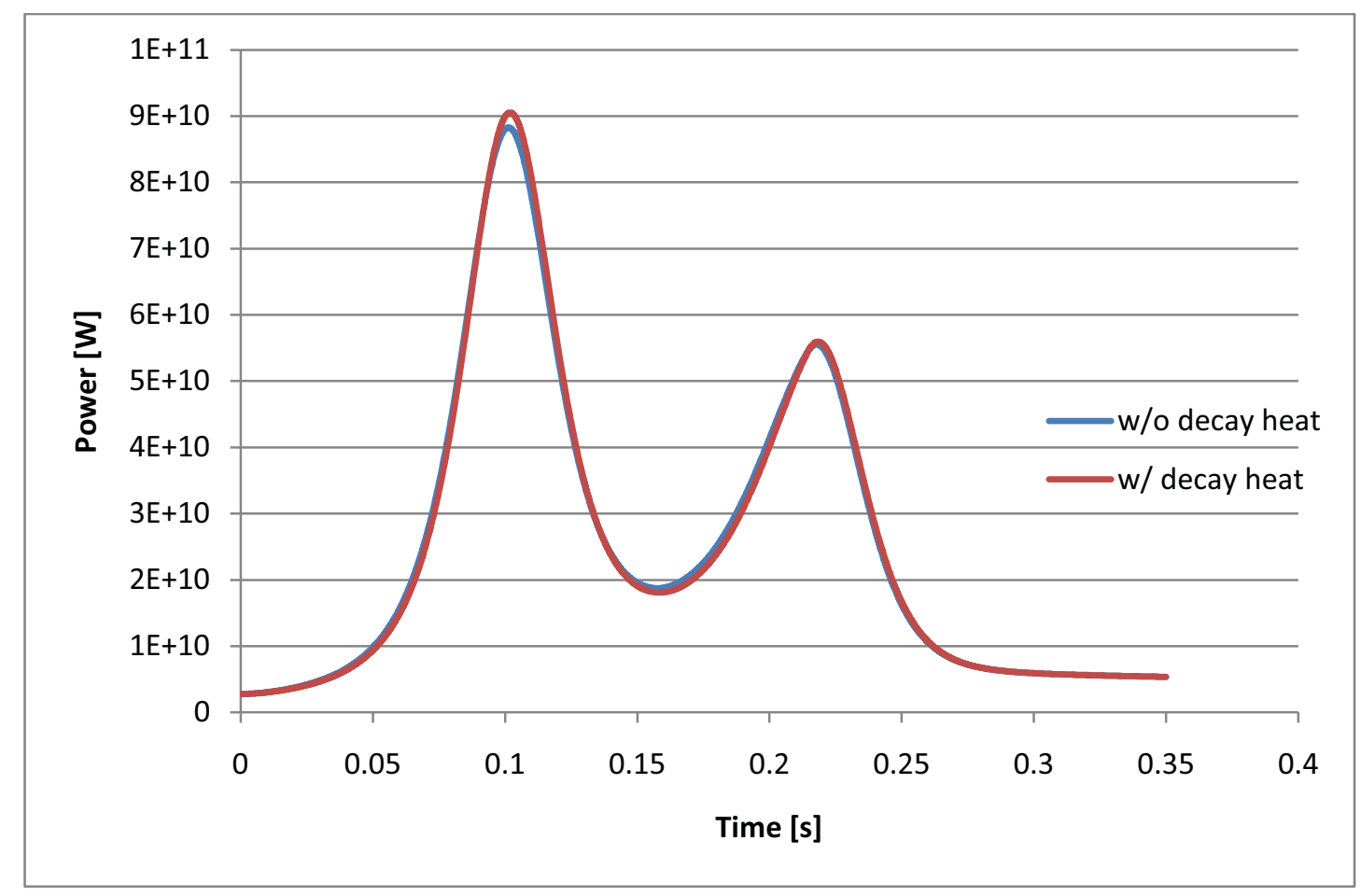

Figure 4. This graph compares the power output of the model with decay heat to the power output when decay heat is ignored. 
Further insight can be gained by calculating the percent difference between the power output. The percent difference between two parameters is calculated using the following equation:

$$
\text { percent difference }=100 * \frac{\left(A_{d h}-A\right)}{A}
$$

where $A$ is the quantity being compared. The $d h$ subscript indicates that the quantity is taken from the model that included decay heat while the absence of a subscript indicates the quantity was taken from the model that did not. Figure 5 shows percent difference for power and fuel temperature.
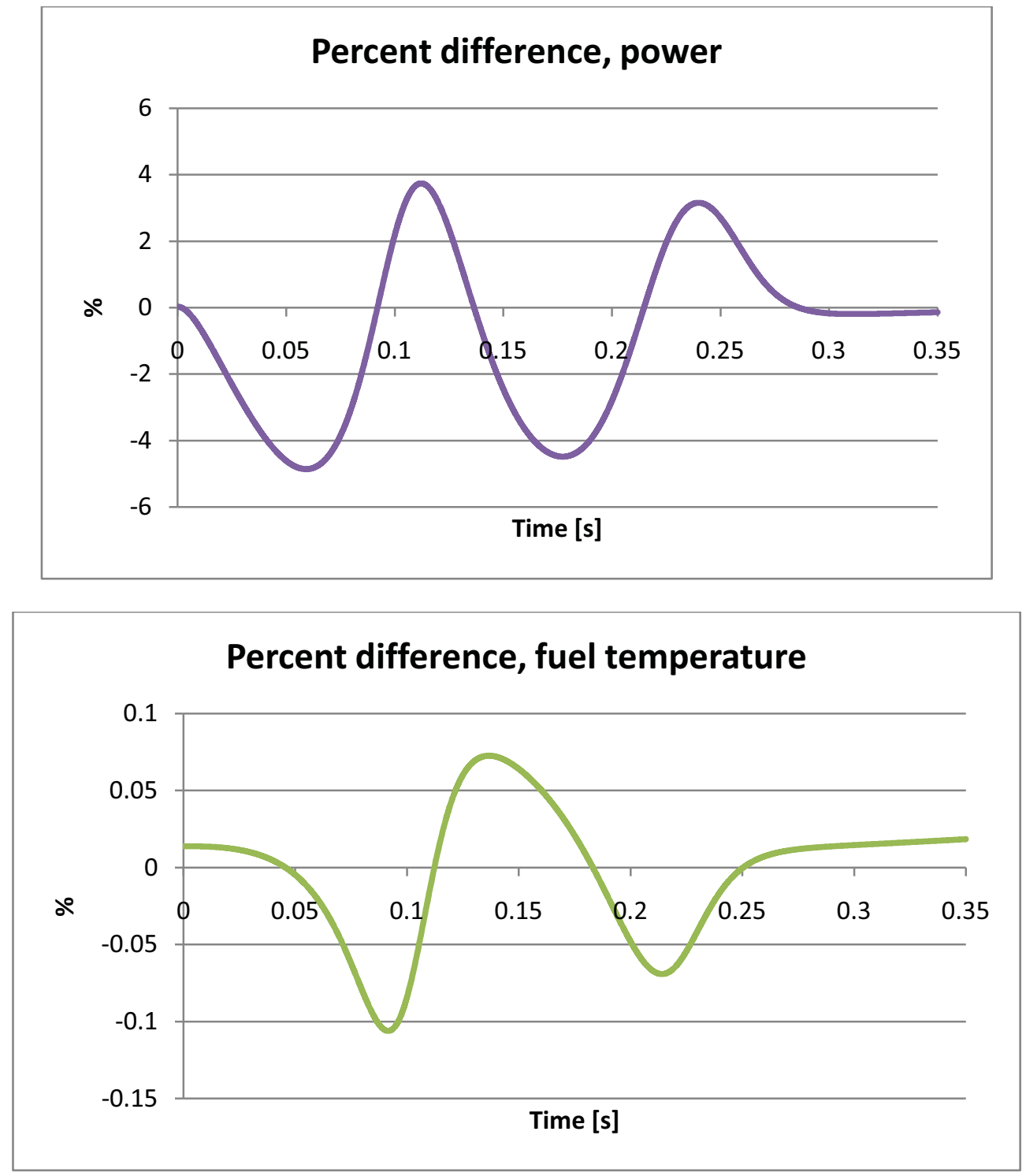

Figure 5. These graphs show the percent difference in power and fuel temperature. 


\section{External Neutron Sources}

While the effects of external neutron sources are negligible when the reactor is operating at any level above zero power, they should still be tracked because of their importance at low power levels and after the reactor has been shutdown. For the purposes of this paper, an external neutron source is any neutron source other than fission.

The obvious external neutron source is a neutron source that has been put into the core to provide neutron detector readings during startup. These can easily be user-specified.

Other neutron sources include spontaneous fission, $(\alpha, n)$ reactions, and photoneutrons. Currently, the ones coded are neutrons generated from spontaneous fission and $(\alpha, n)$ reactions. Other sources, such as photoneutrons, are too small to be of significance in a LWR. Since the code already calculates the actinide inventory, Table III can be used to calculate the neutron source due to spontaneous fission.

\section{Table III.}

This table gives information on the neutron emission rates of several spontaneously fissioning nuclides [18].

\begin{tabular}{|l|l|}
\hline & {$[\mathrm{n} / \mathrm{g}-\mathrm{s}]$} \\
\hline $\mathrm{U}-235$ & $7.43 \mathrm{E}-04$ \\
\hline $\mathrm{U}-238$ & $1.80 \mathrm{E}-02$ \\
\hline $\mathrm{Pu}-238$ & $2.64 \mathrm{E}+03$ \\
\hline $\mathrm{Pu}-239$ & $2.30 \mathrm{E}-02$ \\
\hline $\mathrm{Pu}-240$ & $8.34 \mathrm{E}+02$ \\
\hline $\mathrm{Pu}-242$ & $1.80 \mathrm{E}+03$ \\
\hline $\mathrm{Cm}-242$ & $1.80 \mathrm{E}+07$ \\
\hline $\mathrm{Cm}-244$ & $1.17 \mathrm{E}+07$ \\
\hline
\end{tabular}

In order to calculate the neutron source due to $(\alpha, n)$ reactions, Table IV was used along with the calculated actinide inventory and known actinide decay constants. Since alpha particles do not penetrate very far, using data based only on $\mathrm{UO}_{2}$ should be sufficient. Furthermore, initial results showed that the neutron source due to spontaneous fission was several orders of magnitude larger than the $(\alpha, n)$ source making the neutron source from $(\alpha, n)$ reactions irrelevant. 


\section{Table IV.}

This table shows integral $(\alpha, n)-N e u t r o n$ yields for reactions in $\mathrm{UO}_{2}[19]$.

\begin{tabular}{|l|l|}
\hline & {$\left[10^{-8} \mathrm{n} / \alpha\right]$} \\
\hline $\mathrm{Cm}-244$ & 2.48 \\
\hline $\mathrm{Cm}-242$ & 3.06 \\
\hline $\mathrm{Am}-241$ & 1.97 \\
\hline $\mathrm{Pu}-242$ & 1.45 \\
\hline $\mathrm{Pu}-240$ & 1.72 \\
\hline $\mathrm{Pu}-239$ & 1.71 \\
\hline $\mathrm{Pu}-238$ & 1.98 \\
\hline
\end{tabular}

As a final word of caution, in order to make the units of Equation (5) consistent, the neutron source term $Q$ must have units of scalar flux per second. All data on neutron sources given in this report are neutron emission rates that must be adjusted to flux emission rates. Without knowledge of the source spectra, this can only be done by assuming a neutron speed.

\section{Solution strategy}

After accounting for the point kinetics equations, the fuel depletion equations, the decay heat equations, and the reactor control equations, the result is a system of 110 ODEs. These resulting equations were converted to matrix format and solved as a single system of equations within Matlab using the ODE solver 'ode15s'.

The resulting system of equations is nonlinear. For example, consider Equation (5), the differential equation describing the scalar flux. An abbreviated version of this equation is reproduced below.

$$
\frac{d \phi}{d t}=\frac{\rho(t)+\alpha_{F}\left(T_{F}(t)-T_{F, 0}\right)+\alpha_{M}\left(T_{M}(t)-T_{M, 0}\right)-\beta_{m i x}(t)}{\Lambda} \phi(t)+\text { other terms }
$$

The coefficient of $\phi(t)$ contains reactivity $\rho(t)$, fuel temperature $T_{F}(t)$, and moderator temperature $T_{M}(t)$. In the current simplified model, $T_{M}(t)$ is for most purposes a constant and does not introduce any nonlinearity; however, in a more detailed system the moderator temperature would itself be a non-linear function of $\phi(\mathrm{t})$. The fuel temperature also appears in the coefficient of $\phi(t)$. The Equation (9) describes $T_{F}(t)$ and shows that the fuel temperature depends on the reactor power and, therefore, the scalar flux. Finally, the coefficient contains $\rho(t)$. Reactivity is related to $k_{\text {eff }}$ through Equation (25). Equation (21) shows that $k_{\text {eff }}$ is a 
function of the entire fuel vector, and it is the scalar flux that drives the changes in the fuel vector. The fuel vector changes slowly except for the fission products. The most important fission product with respect to poisoning is ${ }^{135} \mathrm{Xe}$ which responds to changes in $\phi(t)$ with a timescale on the order of hours.

Shampine and Reichelt give an overview of Matlab's ODE solvers in their paper "The Matlab ODE Suite" [20]. Matlab's ode15s solver was selected because the reactor kinetics equations are known to be stiff. The ode15s solver is able to efficiently solve stiff problems though the use of modified BDFs called numerical differentiation formulae (NDFs). Matlab's NDFs are more accurate than the BDFs, but at higher orders are slightly less stable. For each time-step, the newtime value of the solution is solved for using chord iteration. If user-defined error tolerances cannot be met, the solver will adjust the time step size and re-attempt to find a solution. If desired, the user can set the maximum order of the formulae used and whether to use the BDFs instead of the NDFs.

\section{Modeling the Fuel Cycle}

For the purposes of this project, a simplified model of the typical PWR fuel cycle was developed [21]. It was noted that there is considerable operational maneuvering that goes on during the startup of a reactor. Additionally, there can be maneuvering that occurs after the reactor has been brought up to power, for reasons including flux maps, turbine rolls, and the need to perform maintenance on power plant equipment. Such maneuvering removes the reactor system from steady state, inducing changes on various time scales throughout the entire power plant and necessitating a transient analysis of the entire system. This necessitates the development a neutronics module that can run throughout an entire fuel cycle and model unexpected transients that can occur at any time during operation. Incidents may occur during off-normal conditions, so having a robust neutronics module that can predict what happens during off-normal operation is beneficial.

The actual startup of a nuclear reactor is a complex process that can take a week or more. This process was simplified down to a set of reactor maneuvers that captured the major features of a reactor startup. Simplifications had to be made both because the underlying equations are simple models which to do not model the thermal hydraulic behavior of the core and because writing the control logic to govern complex maneuvers is beyond the scope of this report.

In brief, a startup consists of three actions. First, the moderator temperature is brought up to hotstandby by running the reactor coolant pumps. In the current model, the time rate of change of $\mathrm{T}_{\mathrm{M}}$ is given by the user, so modeling this stage is trivial if a representative rate of temperature increase is known. 
The next stage is to bring the reactor up to critical, though both rod movements and boron dilution. 
Table $\mathbf{V}$ lists an abbreviated set of actions required for the startup maneuvers. In the current iteration of the control module, the criticality search is performed by adjusting the boron concentration. Because $\rho$ appears explicitly in the point kinetics equations, moving the reactor to critical is as simple as adjusting the boron concentration until $\rho$ goes as close to zero as numerically feasible. Once the reactor is critical, the neutron population is allowed to grow, usually exponentially, until the reactor reaches two or three percent power. The reactivity insertion necessary to do this is approximated by using the Inhour equation. Because it is only at these very low power levels that exponential growth of the neutron population is allowed, the effects of temperature feedback can be neglected and the Inhour equation can be used in its traditional form. Without feedback, a constant positive reactivity will cause the neutron population to rise exponentially with a stable period. More precisely, the power rise will asymptotically approach a stable period. The asymptotic stable period is reasonably approached somewhere on the order of seconds, so using the stable period as an estimate for reactor control is acceptable.

At low power, the external neutron source can have a large impact on the kinetic behavior of the system and must be accounted for. However, this does not complicate the solution of the problem as the external source represents a particular solution which is added onto the homogenous solution that is generated with the the Inhour equation.

The rest of the control maneuvers used to bring the reactor up to power are either linear power increases or periods of constant power. As the reactor moves up in power, the external sources of neutrons become unimportant and the effects of fuel temperature feedback come into play. In the interest of creating a control algorithm that is flexible and not dependent on the analytical solution of kinetic equations, the currently implemented linear power algorithm uses estimations. A more exact description of the algorithm is given in Appendix A. What the algorithm does, in basic terms, is estimate the reactivity needed by examining the rate of change of power, $d P / d t$, that resulted from the value of reactivity over the last time step. In reality, $d P / d t$, and therefore $d n / d t$, is a function of both the neutron density $n$ and reactivity. Looking at the relationship between the rate of power change and reactivity over only the previous time step has the side effect of encapsulating this dependence (by using the "old-time" value of $n$ to predict $d P / d t$ over the next time step). Once the target value of the reactivity $\rho$ has been determined, the algorithm estimates the external reactivity insertion necessary to reach the required value. This is done by comparing how much external reactivity was inserted last time step to how much the total reactivity changed during the last time step. 
Table V.

Simplified startup procedure

\begin{tabular}{|l|l|}
\hline Action & Time \\
\hline $\begin{array}{l}\text { Heat up coolant from ambient } \\
\text { to hot zero }\end{array}$ & $10[\mathrm{~h}]$ \\
\hline Dilute boron to critical & $\sim 1[\mathrm{~h}]$ \\
\hline $\begin{array}{l}\text { Go to 3\% power at a rate of } \\
\text { half a decade per minute }\end{array}$ & $\sim 1[\mathrm{~h}]$ \\
\hline Go to $8 \%$ power in half a day & $8[\mathrm{~h}]$ \\
\hline Steady state & $1[\mathrm{~h}]$ \\
\hline Go to 20\% power & $1[\mathrm{~h}]$ \\
\hline Steady state & $2[\mathrm{~h}]$ \\
\hline Go to 50\% power & $3[\mathrm{~h}]$ \\
\hline Steady state & $2[\mathrm{~d}]$ \\
\hline Go to $90 \%$ power & $10[\mathrm{~h}]$ \\
\hline Steady state & $1[\mathrm{~d}]$ \\
\hline Go to $100 \%$ power & $10[\mathrm{~h}]$ \\
\hline & \\
\hline Cumulative time & $5[\mathrm{~d}]$ \\
\hline
\end{tabular}

Immediately after trip, the decay heat and the rate at which the neutron population decays become the two most relevant reactor behavior quantities. The rate at which the neutron population decays will quickly become dominated by the longest lived precursor group. Because of this, the accuracy of the point kinetics equations in this situation is very sensitive to the accuracy of the smallest precursor decay constant $\lambda_{i}$. Anything that might introduce additional error into the decay constants, such as refitting delayed neutron data to a consistent set of $\lambda$, should be avoided. Fortunately, the variation between the smallest precursor decay constants for ${ }^{235} \mathrm{U}$ and ${ }^{239} \mathrm{Pu}$ is within the experimental error of the measurement. Thus the amount of error introduced by using a consistent set of decay constants should be insignificant. The other point of interest is whether or not using more delayed neutron groups, specifically a set with more longer lived groups, would give better accuracy over long times [22]. 


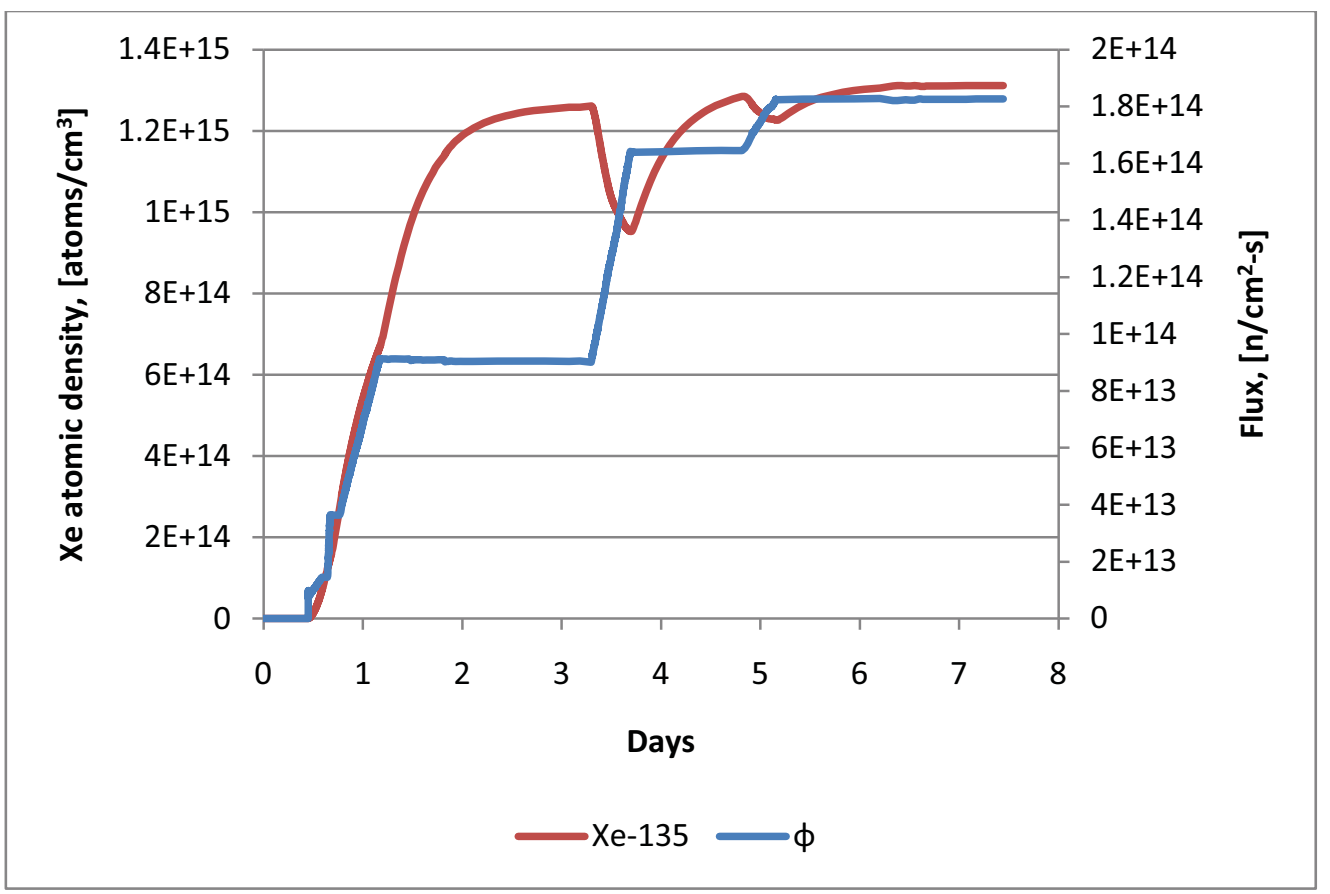

Figure 6. ${ }^{135} \mathrm{Xe}$ buildup responding to the power changes during a reactor startup.

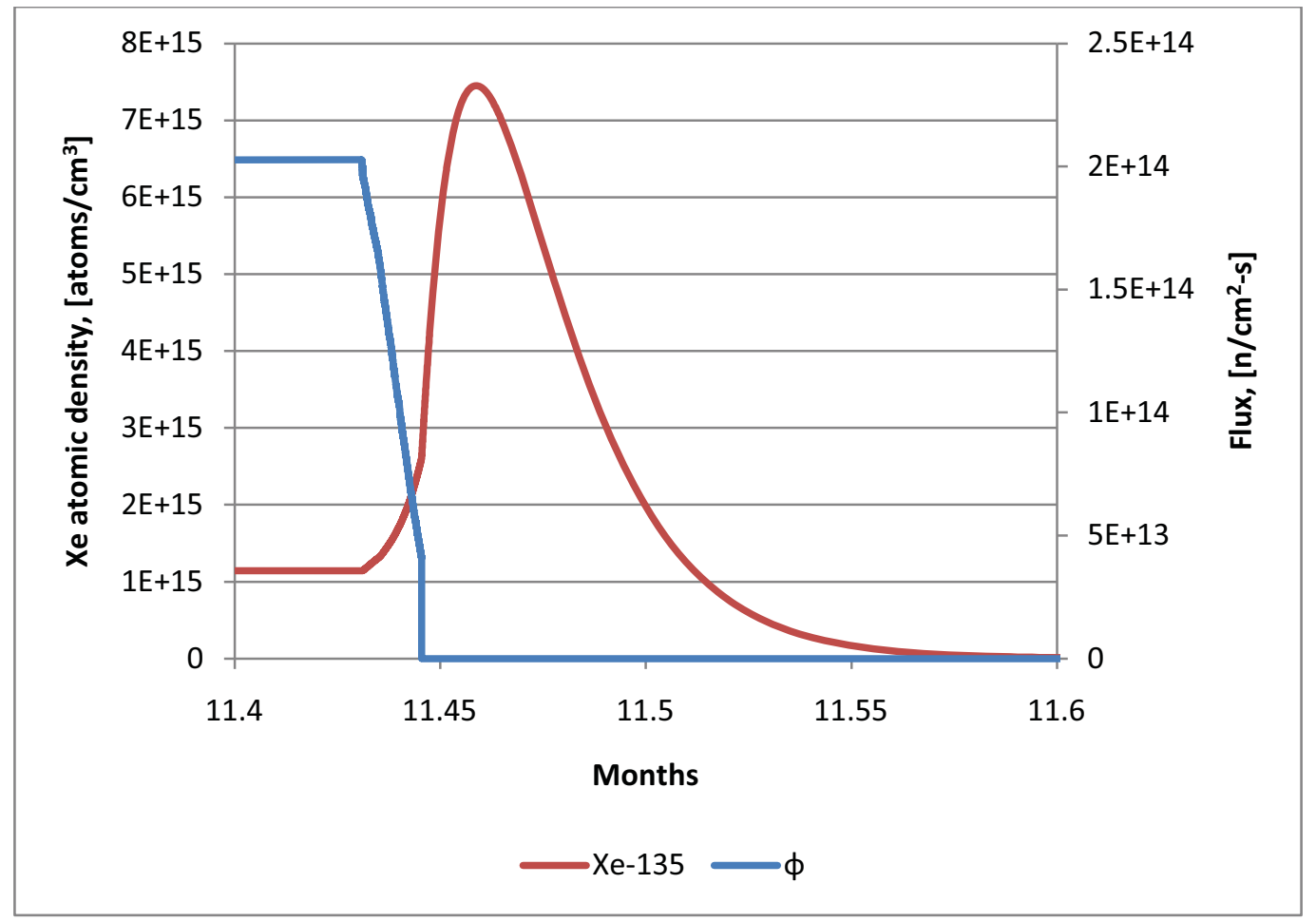

Figure 7. Once the reactor begins down-powering, ${ }^{135} \mathrm{Xe}$ starts to build up. 
After most of the neutron flux has decayed away, the point kinetics equations reduce to subcritical equilibrium. Subcritical equilibrium is achieved as long as there is an external neutron source in the reactor. As discussed above, there is always a neutron source of some magnitude due to spontaneous fission and other atomic interactions. Because the changes are small; we can add the seven point reactor kinetics equations together, and by simplifying and setting the time derivatives to zero, we can use the following relation [1].

$$
0=\frac{\rho(t)}{\Lambda} n(t)+Q(t)
$$

This equation allows for the subcritical equilibrium neutron density to be solved for in terms of , , and $\mathrm{Q}(\mathrm{t})$.

However, because shutdown is not a regime where point kinetics is usually applied, one needs to look at the terms in Equation (34) very closely. First, consider the neutron generation time, $\Lambda$. Like almost any average-like value in nuclear engineering, the rigorous way to calculate the neutron generation time involves weighting over the adjoint flux. However, due to the complexity involved in calculating the adjoint, this method is not typically used. The traditional way to calculate is shown through the following progression of equations

$$
\Lambda_{\infty}=\frac{1}{v \Sigma_{a}}
$$

Equation (35) is the average time it takes for a neutron to be absorbed ( is neutron speed).

$$
\Lambda_{0}=\frac{\Lambda_{\infty}}{\left(1+L^{2} B^{2}\right)}
$$

Equation (36) is the average time it takes for a neutron to be absorbed or for it to leak.

$$
\Lambda=\frac{\Lambda_{0}}{k_{e f f}}
$$

Equation (37) yields the final expression for neutron generation time. However, it is important to note that the neutron generation time depends on the multiplication factor. In the current model,

is set as a constant value. It may be necessary to vary $\Lambda$ with time as $k_{\text {eff }}$ for a shutdown reactor may be considerably less than unity.

Modeling refueling in a zero dimensional model is done by adjusting the isotopic concentrations of the fuel vector. Under the assumption that one-third of the core is replaced with fresh fuel, the concentration of each isotope is adjusted accordingly.

$$
N_{i}^{\text {new }}=\frac{2}{3} N_{i}^{\text {old }}+\frac{1}{3} N_{i}^{\text {fresh }}
$$


In Equation (38), the $i$ subscript represents an element of the fuel vector. The new superscript represents the new fuel vector, the old superscript represents the old, pre-reload fuel vector, and the fresh superscript represents the fresh fuel vector. For commercial $U$ fuel, all of the elements of the fresh fuel vector will be zero except for ${ }^{235} \mathrm{U}$ and ${ }^{238} \mathrm{U}$. In addition, $F$ (see Eq. (24)), the value tracking the total fissions that have occurred in the fuel, must also be reduced by one-third. Once the reactor has been refueled, it is ready for another startup.

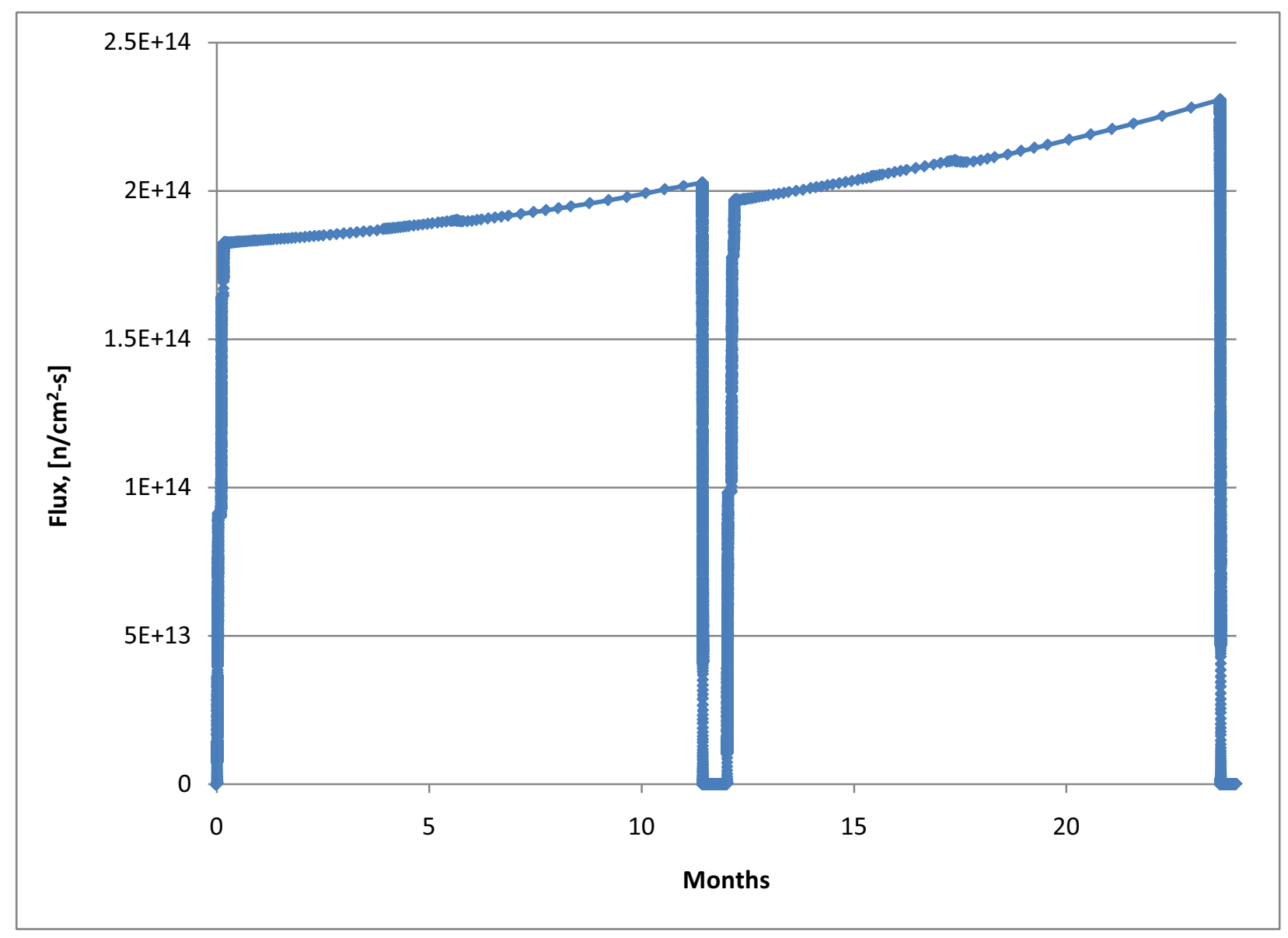

Figure 8. These results were generated by running the code for two consecutive fuel cycles. The reactor operated at the same power level for both cycles. The first cycle was done with completely fresh fuel which caused the flux during this cycle to be lower. The data points are included on the graph to show the varying size of the time steps taken by Matlab's ODE solver. 


\section{Future Work}

The kinds of future work that need to be performed fall into two broad categories, the first of which is work that still needs to be done on the existing point kinetics model. Right now the feedback coefficients, $\alpha_{\mathrm{F}}$ and $\alpha_{\mathrm{M}}$, are constants. The fuel temperature feedback coefficient captures the effects of fuel expansion and Doppler absorption. The Doppler coefficient is a function of fuel temperature as well as fuel composition. Since the isotopic composition of the fuel is already being tracked, adjusting the Doppler coefficient to account for Pu buildup is possible. The moderator temperature coefficient should be a function of both moderator temperature and boron concentration. For example, at high boron concentrations that are typical of a PWR at beginning-of-life, the moderator temperature coefficient can be positive.

The thermal conductivity of the fuel is especially important for transient analysis. The simplified heat transfer model currently implemented in the code does not contain a parameter explicitly modeling the thermal conductivity of the fuel. Nonetheless, capturing how heat transfer changes with burnup should be present in code whose goal is to be able to model transients that occur at any time during reactor operation [23].

The second category of future work will be validating when using the point kinetics model is viable. Due to the computational requirements associated with time dependent transport and diffusion calculations combined with the long length of some accidents (not to mention the length of a full 18-month cycle), it will be beneficial to use point kinetics models as much as possible [24]. 


\section{References}

[1] D.L. Hetrik, Dynamics of Nuclear Reactors. American Nuclear Society, La Grange Park, Illinois, USA (1993).

[2] G.I. Bell and S. Glasstone, Nuclear Reactor Theory. Van Nostrand Reinhold, New York, New York (1970).

[3] J. Duderstadt and L. Hamilton, Nuclear Reactor Analysis. John Wiley \& Sons, New York (1976).

[4] Technology and Applied R\&D Needs for Advanced Nuclear Energy Systems. Resource Document for the Workshop on Basic Research Needs for Advanced Nuclear Energy Systems. DOE Office of Science, June 2006.

[5] G.R. Keepin, Physics of Nuclear Kinetics. Addison-Wesley, Reading, Massachusetts (1965).

[6] A.F. Henry, Nuclear-Reactor Analysis. MIT Press, Cambridge, Massachusetts (1976).

[7] K.O. Ott and R.J. Neuhold, Nuclear Reactor Dynamics. American Nuclear Society, La Grange Park, Illinois, USA (1985).

[8] S. Tashakor, G. Jahanfarnia, and M. Hashemi-Tilehnoee. Numerical solution of the point kinetics equations with fuel burn-up and temperature feedback. Annals of Nuclear Energy, 37 (2010).

[9] http://www.ornl.gov/sci/origen-arp/origen-arp.pdf

[10] A. Tobias, Decay Heat. Progress In Nuclear Energy, 5 (1980).

[11] V.E. Schrock, Evaluation of Decay Heating In Shutdown Reactors. Progress in Nuclear Energy, 3 (1979).

[12] K. Tasaka, et al. JNDC Nuclear Data Library of Fission Products. Japan Atomic Energy Research Institute. JAERI 1287 (1983).

[13] I. Gauld, "Validation of ORIGEN-S Decay Heat Predictions for LOCA Analysis," in Proc. of PHYSOR-2006, American Nuclear Society Topical Meeting on Reactor Physics: Advances in Nuclear Analysis and Simulation, September 10-14, 2006, Vancouver, British Columbia, Canada.

[14] M.P. Paulsen, et al, RETRAN-3D - A Program for Transient Thermal-Hydraulic Analysis of Complex Fluid Flow Systems, Volume 1: Theory and Numerics.

[15] TRAC-B Code Manual 
[16] ANS Standards Working Group, "ANS-5.1: Decay Heat Power in Light Water Reactors," ANS, ANSI/ANS-5.1-1979, August 1979.

[17] J.R. Lamarsh, Introduction to Nuclear Engineering. Addison-Wesley, Reading, Massachusetts (1983).

[18] A. Lorenz, A Survey of Neutron Sources and Their Applications. UCRL-51298. Lawrence Livermore Laboratory, (1972).

[19] H.W. Wiese, Neutron Source and Spectra from $(\alpha, n)$-Reactions and Spontaneous Fission in Spent Fuel and Vitrified High Active Waste. NEACRP-L-278. Nuclear Research Center, Karlsruhe, Germany.

[20] L.F. Shampine, and M.W. Reichelt, "The MATLAB ODE Suite," SIAM Journal on Scientific Computing, 18 (1997).

[21] B. Phoenix, private communication, 2010.

[22] G.D. Spriggs, J.M. Campbell, and V.M. Piksajkin, An 8-Group Delayed Neutron Model Based on a Consistent Set of Half-Lives. Progress in Nuclear Energy, 41 (2002).

[23] S.G. Popov, J.J. Carbajo, V.K. Ivanov, G.L. Yoder, Thermophysical Properties of MOX and $\mathrm{UO}_{2}$ Fuels Including the Effects of Irradiation. Oak Ridge National Laboratory, Engineering Technology Division, ORNL/TM-2000/351 (2000).

[24] D.G. Cacuci, Dimensionally adaptive dynamic switching and adjoint sensitivity analysis: new features of the RELAP5/PANBOX/COBRA code system for reactor safety transients. Nuclear Engineering and Design, 202 (2000). 


\section{Appendix A - Control Algorithms}

The control algorithm, contained in the function control_module, is called after the successful solution of each time step.

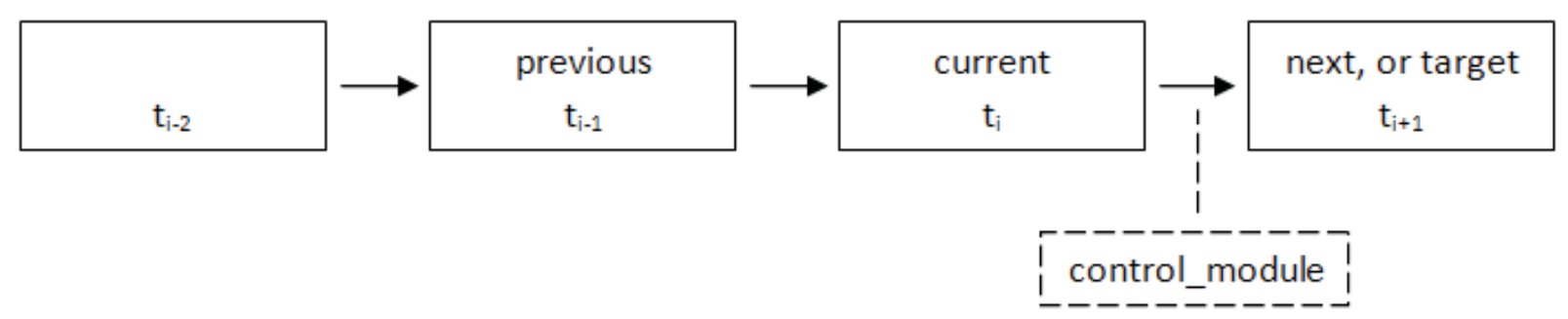

Figure A-1. This graphic illustrates when control_module is called and what terms are used in this appendix to describe the time steps before and afterwards.

\section{Criticality search algorithm}

First, the change in reactivity necessary to bring the reactor critical is determined.

$$
\Delta \rho=\rho_{\text {target }}-\rho_{\text {current }}
$$

Since criticality is desired, $\rho_{\text {target }}$ will be equal to zero. The change in boron concentration needed to effect $\Delta \rho$ is found using Equation 2 .

$$
\Delta B=\frac{\Delta \rho}{\text { differential boron worth }}
$$

Here, $\Delta \rho$ the target change in reactivity calculated in Equation 1 is divided by the differential boron worth, $\frac{\partial \rho}{\partial \text { boron concentration }}$. The result, $\Delta B$, is the desired change in boron concentration. The target boron concentration is given by Equation 3 .

$$
B_{\text {target }}=B_{\text {current }}+\Delta B
$$

The boron concentration is then adjusted until the target boron concentration is reached.

$$
\frac{d \text { boron }}{d t}=\left(\frac{d \rho}{d t}\right)_{\text {target }} * \frac{\partial(\text { boron concentration })}{\partial \rho}
$$


Equation 4 shows that the boron concentration is adjusted in order to introduce reactivity at the

rate given by $\left(\frac{d \rho}{d t}\right)_{\text {target }}$. In the current reactor model, $\frac{\partial \rho}{\partial \text { boron concentration }}$ is constant, so the rate of change of the boron concentration will also be constant.

\section{Exponential power rise algorithm}

The exponential power rise algorithm is based on the solution to the Inhour equation. The time dependent neutron population is approximately described by the following relation

$$
n(t)=n_{0} e^{\alpha t}+Q t
$$

where $n(t)$ is the time dependent neutron population, $n_{0}$ is the neutron population at an initial reference time, $\alpha$ is the stable reactor period, and $Q$ is that rate at which an external source is introducing neutrons into the system. Rearranging Equation 5 yields:

$$
\alpha=\frac{1}{t} \ln \left(\frac{n(t)-Q t}{n_{0}}\right)
$$

Since exponential growth is desired, $n(t)$ can be replaced by $A n_{0}$, where $A$ is a constant that determines the rate of growth. This substitution is shown in Equation 7.

$$
\alpha=\frac{1}{t} \ln \left(\frac{A n_{0}-Q t}{n_{0}}\right)
$$

Equation 8 can then be solved for the stable reactor period. Once the desired stable reactor period is known, the reactivity needed to induce this is found using the Inhour formula.

$$
\rho_{\text {target }}=\alpha \Lambda+\sum_{k} \frac{\beta_{k} \alpha}{\alpha+\lambda k}
$$

With the target reactivity known, the target rod bank position necessary to induce the target reactivity is found by interpolating the table containing rod worth and position. The control rods are adjusted until the target position is reached.

$$
\frac{d r o d}{d t}=\left(\frac{d \rho}{d t}\right)_{\text {target }} * \frac{\partial(\text { rod })}{\partial \rho}
$$




\section{Linear power rise algorithm}

Many reactor maneuvers require that the reactor power level change as a linear function of time. Due to the feedback mechanisms and nonlinearities present in the reactor kinetics and dynamics equations, an inexact control algorithm was developed.

The first step is to numerically evaluate the current time rate of change of the power.

$$
\begin{gathered}
\left(\frac{d P}{d t}\right)_{\text {current }}=\frac{P_{i}-P_{i-1}}{t_{i}-t_{i-1}} \\
\left(\frac{d P}{d t}\right)_{\text {previous }}=\frac{P_{i-1}-P_{i-2}}{t_{i-1}-t_{i-2}}
\end{gathered}
$$

Next $\left(\frac{d P}{d t}\right)_{\text {current }},\left(\frac{d P}{d t}\right)_{\text {previous }}, \rho_{\text {current }}$, and $\rho_{\text {previous }}$ are compared and linear extrapolation is performed to determine $\rho_{\text {next }}$, the target reactivity to be inserted over the next time step. This is shown in Equations 12 and 13.

$$
\begin{gathered}
m=\frac{\left(\rho_{\text {current }}-\rho_{\text {previous }}\right)}{\left({\frac{d P}{d t_{\text {current }}}}-\frac{d P}{d t_{\text {previous }}}\right)} \\
\rho_{\text {target }}=m\left(\frac{d P}{\left.d t_{\text {target }}-\frac{d P}{d t}_{\text {current }}\right)+\rho_{\text {current }}}\right.
\end{gathered}
$$

Once the target reactivity is known, the next step is to calculate $\Delta \rho_{\text {target }}$, the target change in reactivity to be made over the next time step.

$$
\Delta \rho_{\text {target }}=\rho_{\text {target }}-\rho_{\text {current }}
$$

The change in reactivity over a time step can be expressed as the sum of the change in reactivity introduced by external control mechanisms, the change in reactivity introduced by feedback, and the change in reactivity introduced by other causes (for example burnup).

$$
\Delta \rho_{\text {total }}=\Delta \rho_{\text {ex }}+\Delta \rho_{\text {feedback }}+\Delta \rho_{\text {other }}
$$

First, the total reactivity change over the previous time step is calculated

$$
\Delta \rho_{\text {total }}=\rho_{\text {current }}-\rho_{\text {previous }}
$$


The change in reactivity due to control mechanisms is given by the following equation

$$
\Delta \rho_{\mathrm{ex}}=\left(R_{\text {current }}-R_{\text {previous }}\right)+\left(B_{\text {current }}-B_{\text {previous }}\right) * \frac{\partial \rho}{\partial B}
$$

where $\mathrm{R}$ is the worth of the control rods and $B$ is the boron concentration. The change in reactivity due to Doppler feedback is given by Equation 18.

$$
\Delta \rho_{\text {feedback }}=\alpha_{T}\left(T_{F, \text { current }}-T_{F, \text { previous }}\right)
$$

Since $\Delta \rho_{\text {other }}$ could be caused by any number of things, it is calculated using Equation 19.

$$
\Delta \rho_{\text {other }}=\Delta \rho_{\text {total }}-\Delta \rho_{\text {ex }}-\Delta \rho_{\text {feedback }}
$$

Equations 17 though 19 all dealt with values of $\Delta \rho$ over the previous time step. However, the goal of the control algorithm is to predict what these values will be over the next time step.

Once this has been done, it will be necessary to determine what $\Delta \rho_{\mathrm{ex}}$ will be necessary over the next time step to reach the target $\Delta \rho_{\text {total }}$. Looking at Equation 16, we see that in order to estimate $\Delta \rho_{\text {ex }}$ over the next time step, we need to estimate the values $\Delta \rho_{\text {feedback }}$ and $\Delta \rho_{\text {other }}$ for the next time step. $\Delta \rho_{\text {other }}$ is estimated using old-time value as calculated in Equation Error! Reference source not found., but $\Delta \rho_{\text {feedback }}$ is estimated by using linear extrapolation to determine the value of $T_{F}$ at the end of the next time step.

$$
m_{T_{F}}=\frac{\left(T_{F, \text { current }}-T_{F, \text { previous }}\right)}{\left(\frac{d P}{d t_{\text {current }}}-\frac{d P}{d t_{\text {previous }}}\right)}
$$

Then

$$
T_{\mathrm{F}, \text { target }}=m_{T_{F}}\left(\frac{d P}{d t}_{\text {target }}-\frac{d P}{d t}{ }_{\text {current }}\right)+T_{\mathrm{F}, \text { current }}
$$

Putting everything together yields the final relation for the change in external reactivity that needs to be effected over the next timestep.

$$
\Delta \rho_{\mathrm{ex}}^{\mathrm{target}}=\Delta \rho_{\mathrm{target}}-\alpha_{T_{F}}\left(T_{F}^{\mathrm{target}}-T_{F}^{\mathrm{current}}\right)-\Delta \rho_{\mathrm{other}}^{\text {current }}
$$

The rate of change or rods, boron, or some combination of both can then be determined from Equation 23. As a word of caution, there is no way for control_module to know the size of the next time step $(\Delta t)$ so the size of the previous time step must be used instead. 


$$
\Delta \rho_{\mathrm{ex}}^{\mathrm{next}}=\left(\frac{d r o d}{d t} \frac{\partial \rho}{\partial \operatorname{rod}}+\frac{d B}{d t} \frac{\partial \rho}{\partial B}\right) \Delta t
$$

\section{(Pseudo) Steady state algorithm}

The steady state algorithm is simply the linear power rise algorithm with $\frac{d P}{d t}$ set to zero. Because of the changes in reactivity that occur as a result of burn up, control adjustments need to be made to hold the reactor at constant power.

\section{Shutdown algorithm}

For standard power decreases, the linear power rise algorithm can be used by inputting a negative value for $\frac{d P}{d t}$ target . The shutdown algorithm is used at the end of a cycle to trip the reactor, bring the boron levels up to refueling concentration, and cool the moderator back to ambient temperature. The rod insertion rate is set to 0.333 , which represents fully out to fully in within 3 seconds. The rate of change of the boron concentration is set so that the boron concentration will go from $0 \mathrm{ppm}$ to $2000 \mathrm{ppm}$ in 24 hours. The rate of change of the moderator temperature is set to make $T_{M}$ go from $580 \mathrm{~K}$ to $300 \mathrm{~K}$ in 24 hours. Once the shutdown algorithm has been called, there is no way to abort it, so it runs until for the remaining irradiation time. Once the $t_{\text {end }}$ is reached, program control returns to main.m where a fuel reload can be performed and ode15s.m called again to start another fuel cycle. 